Proc. XII Int. School on Theoretical Physics — Symmetry and Structural Properties of Condensed Matter

\title{
Thermal Entanglement and Quantum Non-Locality along the Stepwise Magnetization Curve of the Spin-1/2 Ising-Heisenberg Trimerized Chain
}

\begin{abstract}
J. StREČKA* AND J. PAVLIČKO
Institute of Physics, Faculty of Science, P.J. Šafárik University, Park Angelinum 9, 04154 Košice, Slovakia

The spin-1/2 Ising-Heisenberg trimerized chain in a magnetic field is revisited with the aim to explore the quantum entanglement and non-locality within the exactly solved spin system, which exhibits in a low-temperature magnetization curve two intermediate plateaux at zero and one-third of the saturation magnetization. The groundstate phase diagram involves two quantum (antiferromagnetic, ferrimagnetic I) and two classical (ferrimagnetic II, saturated paramagnetic) phases. We have rigorously calculated the concurrence and Bell function in order to quantify the quantum entanglement and non-locality at zero as well as non-zero temperatures. It is demonstrated that the entanglement can be thermally induced also above the classical ground states unlike the quantum nonlocality, which means that the thermal entanglement is indispensable for a violation of the locality principle.
\end{abstract}

DOI: 10.12693/APhysPolA.132.167

PACS/topics: 75.10.Pq, 75.10.Jm, 75.40.Cx, 75.60.Ej

\section{Introduction}

Quantum entanglement has attracted a great deal of attention since early work by Einstein, Podolsky and Rosen (EPR) questioned sustainability of quantum mechanics through one of its most famous paradoxes [1]. Interestingly, EPR paradox helped a discovery of the quantum non-locality since Bell put forward inequality, which is violated by certain set of entangled states [2]. It is worthwhile to remark, however, that the quantum entanglement and non-locality are distinct albeit closely related features of quantum-mechanical systems [3]. Moreover, it has been evidenced that the quantum entanglement and non-locality are also capable of detecting phase transitions of quantum spin systems [4-6].

Quantum effects may have striking macroscopic consequences like for instance presence of quantized plateaux in low-temperature magnetization curves. The magnetization plateaux of quantum spin chains should satisfy Oshikawa-Yamanaka-Affleck rule [7]: $p\left(S_{u}-m_{u}\right)=Z$, where $p$ is the ground-state period, $S_{u}$ and $m_{u}$ is the total spin and total magnetization of elementary unit and $Z$ is an integer number. This rule represents necessary but not sufficient condition for existence of magnetization plateaux and thus, one should verify their actual existence case by case by performing specific calculations.

In the present work, we will revisit the spin- $1 / 2$ IsingHeisenberg trimerized chain, which exhibits the stepwise magnetization curve with two intermediate plateaux at zero and one-third of the saturation magnetization either of quantum or classical nature [8]. Our main goal is to investigate the quantum entanglement and non-locality of this model at zero as well as non-zero temperatures.

*corresponding author; e-mail: jozef.strecka@upjs.sk

\section{Model and method}

The spin-1/2 Ising-Heisenberg trimerized chain, which is schematically illustrated in Fig. 1, can be defined through the following Hamiltonian:

$$
\begin{aligned}
\hat{\mathcal{H}} & =\sum_{k=1}^{N}\left\{J\left[\Delta\left(\hat{S}_{k 1}^{x} \hat{S}_{k 2}^{x}+\hat{S}_{k 1}^{y} \hat{S}_{k 2}^{y}\right)+\hat{S}_{k 1}^{z} \hat{S}_{k 2}^{z}\right]\right. \\
& \left.+J_{1}\left(\hat{S}_{k 1}^{z} \mu_{k}^{z}+\hat{S}_{k 2}^{z} \mu_{k+1}^{z}\right)-H_{A} \mu_{k}^{z}-H_{B}\left(\hat{S}_{k 1}^{z}+\hat{S}_{k 2}^{z}\right)\right\},
\end{aligned}
$$

where $\mu_{k}^{z}= \pm 1 / 2$ are the Ising spins and $\hat{S}_{k \gamma}^{\alpha}(\alpha=$ $x, y, z ; \gamma=1,2)$ stand for spatial components of the spin$1 / 2$ operator of the Heisenberg spins. The first term $J$ accounts for the XXZ Heisenberg interaction between the nearest-neighbor Heisenberg spins ( $\Delta$ is the respective anisotropy parameter), the second term $J_{1}$ represents the Ising interaction between the nearest-neighbor Ising and Heisenberg spins, $H_{A}$ and $H_{B}$ are external magnetic fields acting on the Ising and Heisenberg spins, respectively.

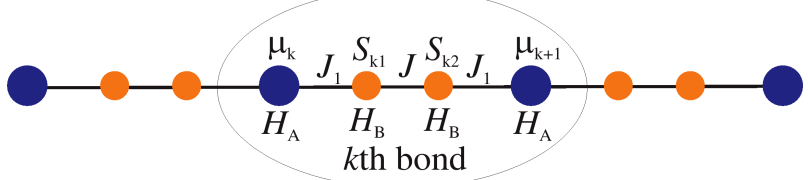

Fig. 1. A schematic representation of the spin- $1 / 2$ Ising-Heisenberg trimerized chain. Large (blue) circles denote lattice positions of the Ising spins and small (orange) circles denote lattice positions of the Heisenberg spins.

It has been shown in our previous work [8] that the generalized decoration-iteration transformation establishes a rigorous mapping correspondence between the spin- $1 / 2$ Ising-Heisenberg trimerized chain given by the Hamiltonian (1) and the effective spin- $1 / 2$ Ising chain, which can 
be further exactly solved by the transfer-matrix method. As a result, one may easily derive exact results for the magnetization and pair correlation functions as given by Eqs. (10),(11) of Ref. [8]. These quantities can be further utilized for a rigorous calculation of the entanglement and non-locality measures.

The bipartite entanglement between the nearestneighbor Heisenberg spins can be quantified at zero as well as non-zero temperatures via the concurrence [9]:

$$
C=\max \left\{0,4\left|q_{h h}^{x x}\right|-2 \sqrt{\left(\frac{1}{4}+q_{h h}^{z z}\right)^{2}-\left(m_{B}^{z}\right)^{2}}\right\},
$$

which can be calculated from the known exact results [8] for the pair correlation functions $q_{h h}^{\alpha \alpha} \equiv\left\langle\hat{S}_{k 1}^{\alpha} \hat{S}_{k 2}^{\alpha}\right\rangle$ $(\alpha=x, z)$ and the magnetization $m_{B}^{z} \equiv\left\langle\left(\hat{S}_{k 1}^{z}+\hat{S}_{k 2}^{z}\right) / 2\right\rangle$. If the concurrence (2) is non-zero, then the system is quantum-mechanically entangled.

The quantum non-locality can be tested through the Horodecki formula [10]:

$$
B=\max \left\{8 \sqrt{2\left(q_{h h}^{x x}\right)^{2}}, 8 \sqrt{\left(q_{h h}^{x x}\right)^{2}+\left(q_{h h}^{z z}\right)^{2}}\right\} \leq 2,
$$

which is widely used form of the Bell inequality derived from the Clauser-Horne-Shimony-Holt inequality [11]. If the inequality (3) is not satisfied, then the system violates the locality principle.

\section{Results and discussion}

In this section, we will perform a comprehensive analysis of the quantum entanglement and non-locality of the spin-1/2 Ising-Heisenberg trimerized chain with the antiferromagnetic interactions $J>0$ and $J_{1}>0$, which is expected to exhibit the most pronounced quantum features. To avoid overparametrization, the local magnetic fields of the Ising and Heisenberg spins will be set equal to $H \equiv H_{A}=H_{B}$ and the Heisenberg coupling will be assumed to be isotropic $(\Delta=1)$.

At first, let us shortly comment on all possible ground states of the model under investigation. The groundstate phase diagram totally involves two quantum and two classical ground states, which can be identified as the quantum antiferromagnetic phase (AF), the quantum ferrimagnetic phase (FI), the classical ferrimagnetic phase (FII) and the classical saturated paramagnetic phase (SP) given by the eigenvectors

$$
\begin{aligned}
& |\mathrm{AF}\rangle=\prod_{k=1}^{N / 2}|\uparrow\rangle_{\mu_{2 k-1}} \otimes \frac{1}{\sqrt{2}}\left(a_{-}|\uparrow \downarrow\rangle-a_{+}|\downarrow \uparrow\rangle\right)_{2 k-1} \\
& \otimes|\downarrow\rangle_{\mu_{2 k}} \otimes \frac{1}{\sqrt{2}}\left(a_{+}|\uparrow \downarrow\rangle-a_{-}|\downarrow \uparrow\rangle\right)_{2 k}, \\
& |\mathrm{FI}\rangle=\prod_{k=1}^{N}|\uparrow\rangle_{\mu_{k}} \otimes \frac{1}{\sqrt{2}}(|\uparrow \downarrow\rangle-|\downarrow \uparrow\rangle)_{k}, \\
& |\mathrm{FII}\rangle=\prod_{k=1}^{N}|\downarrow\rangle_{\mu_{k}} \otimes|\uparrow \uparrow\rangle_{k},|\mathrm{SP}\rangle=\prod_{k=1}^{N}|\uparrow\rangle_{\mu_{k}} \otimes|\uparrow \uparrow\rangle_{k} \cdot(4)
\end{aligned}
$$

In above, the first (second) ket vector determines the spin state of the Ising (Heisenberg) spins from the $k$-th unit cell and the respective probability amplitudes read $a_{ \pm}=\sqrt{1 \pm \frac{J_{1}}{\sqrt{J_{1}^{2}+J^{2} \Delta^{2}}}}$.
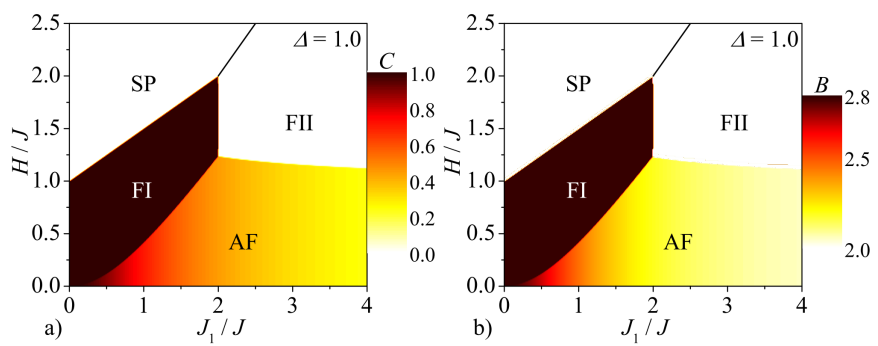

Fig. 2. Zero-temperature density plot of the concurrence (left) and Bell function (right) in the $J_{1} / J-H / J$ plane.

Zero-temperature density plots of the concurrence and Bell function (Fig. 2) calculated for the Heisenberg spin pairs of the trimerized Ising-Heisenberg chain clearly demonstrate abrupt changes of the quantum entanglement and non-locality across all phase-transition lines except the one between two classical ground states FII and SP. It is evident from Fig. 2 that the concurrence and Bell function display quite similar features: (i) they point on absence of quantum correlations $(C=B=0)$ in the classical phases FII and SP; (ii) they reach maximum values $(C=1, B=\sqrt{8})$ in the quantum ferrimagnetic phase FI; (iii) they are gradually suppressed in the quantum antiferromagnetic phase AF upon strengthening of the ratio $J_{1} / J$ between the Ising and Heisenberg interactions according to the formulae:

$$
C=\frac{J \Delta}{\sqrt{J_{1}^{2}+(J \Delta)^{2}}} \quad \text { and } \quad B=2 \sqrt{1+\frac{(J \Delta)^{2}}{J_{1}^{2}+(J \Delta)^{2}}} .
$$

The aforementioned results verify the classical character of the ground states FII and SP, the outstanding quantum entanglement and non-locality in the ground state $\mathrm{AF}$ and the perfect quantum entanglement and non-locality in the ground state FI. It is worthwhile to recall that the zero-temperature magnetization curve of the spin-1/2 Ising-Heisenberg trimerized chain displays two intermediate plateaux at zero and one-third of the saturation magnetization [8], whereas the zero plateau always corresponds to the quantum antiferromagnetic phase AF and the one-third plateau either corresponds to the quantum (FI) or classical (FII) ferrimagnetic phase. In contrast with general expectations, the quantum entanglement and non-locality thus strengthen along the fieldinduced phase transition $\mathrm{AF} \rightarrow \mathrm{FI}$ driven by the increasing magnetic field.

Furthermore, let us examine the concurrence and Bell function as eligible quantifiers of the entanglement and non-locality at finite temperatures. For this purpose, Fig. 3 illustrates density plots of the concurrence and Bell function in the field-temperature plane for two different values of the ratio between the Ising and Heisen- 

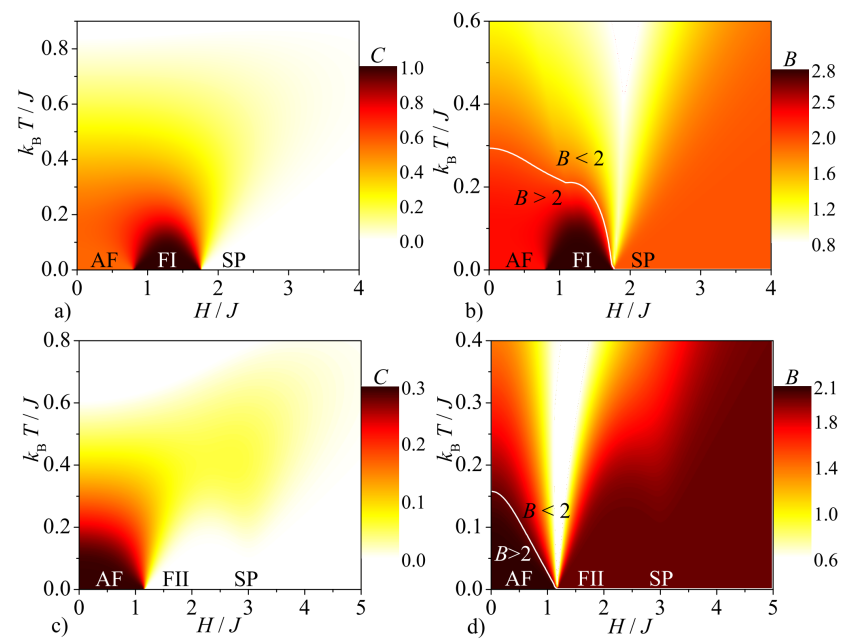

Fig. 3. The density plot of the concurrence (left part) and Bell function (right part) as a function of the magnetic field and temperature by assuming two different values of the ratio: $(\mathrm{a}, \mathrm{b}) J_{1} / J=1.5,(\mathrm{c}, \mathrm{d}) J_{1} / J=3.0$. The locality principle is violated in part (b) and (d) below displayed white lines.

berg interaction $J_{1} / J=1.5$ and 3.0 pertinent to two available magnetization scenarios $\mathrm{AF} \rightarrow \mathrm{FI} \rightarrow \mathrm{SP}$ and $\mathrm{AF} \rightarrow \mathrm{FII} \rightarrow \mathrm{SP}$, respectively. In the former case, the quantum entanglement and non-locality can be temporarily reinforced by the rising magnetic field due to the field-driven phase transition $\mathrm{AF} \rightarrow$ FI emerging at the first critical field $H_{c 1} / J=\sqrt{13} / 2-1 \approx 0.8$, while the second field-driven phase transition $\mathrm{FI} \rightarrow \mathrm{SP}$ at the critical field $H_{c 2} / J=1.75$ is responsible at low enough temperatures for a sudden breakdown of the quantum entanglement and non-locality (see Fig. 3a,b). It should be mentioned that the quantum entanglement contra intuitively intervenes above the classical ground state SP due to a thermal activation of quantum excited states. As a matter of fact, the colored parameter space in Fig. 3a inside of which the concurrence is non-zero corroborates the thermally-induced entanglement intervening above the ground state SP. The similar trend cannot be observed in Fig. 3b in the relevant behavior of the Bell function, which serves as a measure of the quantum nonlocality.

The quantum entanglement and non-locality vanish along the other possible zero-temperature magnetization curve $\mathrm{AF} \rightarrow \mathrm{FII} \rightarrow \mathrm{SP}$ already at the first critical field $H_{c 1} / J=\sqrt{10}-2 \approx 1.2$, which is connected to the field-driven phase transition AF $\rightarrow$ FII (see Fig. 3c,d). Hence, it follows that the quantum entanglement and non-locality do appear at low enough temperatures just above the ground state AF. However, it can be seen from Fig. $3 \mathrm{c}$ that the entanglement can be thermally induced above both classical ground states FII and SP as inferred by a colored parameter region with the non-zero concurrence, which has the shape of a relatively large loop intervening above the ground states FII and SP. This behavior is in sharp contrast to the quantum non-locality, which cannot be thermally activated through the population of quantum excited states.

\section{Conclusions}

In the present work, we have rigorously examined the quantum entanglement and non-locality of the spin$1 / 2$ Ising-Heisenberg trimerized chain at zero as well as non-zero temperatures through the concurrence and Bell function. It has been shown that the entanglement and non-locality measures may serve due to their abrupt changes as good indicators of all field-driven phase transitions except the one between two classical phases. Moreover, it has been evidenced that the entanglement may emerge above classical ground states due to a thermal activation of quantum excited states unlike the quantum non-locality, which appears just above quantum ground states. It could be thus concluded that the entanglement is indispensable for a violation of the locality principle, but the reverse statement does not hold in general.

\section{Acknowledgments}

This work was financially supported under the grants No. VEGA 1/0043/16 and APVV-14-0073.

\section{References}

[1] A. Einstein, B. Podolsky, N. Rosen, Phys. Rev. 47, 777 (1935).

[2] J.S. Bell, Physica 47, 195 (1964).

[3] R.F. Werner, Phys. Rev. A 40, 4277 (1989).

[4] L. Justino, T.R. de Oliveira, Phys. Rev. A $\mathbf{8 5}$, 052128 (2012).

[5] Y.Z. Sun, L. Li, N. Li, K.L. Yao, J. Liu, B. Luo, G.H. Du, H.N. Li, Euro Phys. Lett. 95, 30008 (2011).

[6] B. Wang, H.L. Huang, Z. Sun, S. Kou, Chin. Phys. Lett. 29, 120301 (2012).

[7] M. Oshikawa, M. Yamanaka, I. Affleck, Phys. Rev. Lett. 78, 1984 (1984).

[8] J. Strečka, M. Jaščur, J. Phys. Condens. Matter 15, 4519 (2003).

[9] W.K. Wootters, Phys. Rev. Lett. 80, 2245 (1998).

[10] R. Horodecki, P. Horodecki, M. Horodecki, Phys. Lett. A 200, 340 (1995).

[11] J.F. Clauser, M.A. Horne, A. Shimony, R.A. Holt, Phys. Rev. Lett. 23, 880 (1969). 